\title{
Star formation and black hole accretion at high redshift (review)
}

\section{Hagai Netzer*}

School of Physics and Astronomy, Tel Aviv University, Israel

E-mail: netzer@wise.tau.ac.il

Various ways to probe the co-evolution of super massive black holes and their host galaxies are reviewed and discussed. An integrated approach to the problem, involving the measurement of black holes (BHs) and galaxy masses at all redshifts, is compared with a differential instantaneous way where the observables are the active galactic nucleus (AGN) luminosity, $L_{A G N}$, and the star formation luminosity, $L_{S F}$. The missing ingredients in both methods are the mass function and duty cycle of BHs. I demonstrate how new data on very high redshift objects enable first reliable estimates of the duty cycles of the most massive BHs, and argue that the extreme star formation rates in their host galaxies indicate large mergers. The idea of creating a "BH mass sequence" is examined using data from large AGN surveys. While this is a promising method, most present day data are inadequate for such an analysis because of various selection effects. I present a new attempt to use semi-analytic models to explain new observations of $L_{A G N}$ versus $L_{S F}$ in various samples observed by Herschel. The model indicates that a scenario where $\mathrm{BH}$ accretion is triggered by mergers is in good agreement with observations despite various suggestions that secular evolution plays a more important role in most AGNs.

Nuclei of Seyfert galaxies and QSOs - Central engine \& conditions of star formation November 6-8, 2012

Max-Planck-Insitut für Radioastronomie (MPIfR), Bonn, Germany

\footnotetext{
*Speaker.
} 


\section{Stellar mass black hole mass and co-evolution}

\subsection{The two flavors of co-evolution}

There are two parallel, somewhat different ways to follow the accumulation of black hole $(\mathrm{BH})$ and stellar mass in bulge galaxies, those containing super massive BHs in their centers. The first is an integrated approach, where we follow the total stellar and BH mass through cosmic time. Studying this type of "co-evolution" is a very active area of research related to various types of correlations between BH and stellar mass (Ferrarese and Merritt 2000; Gebhardt et al. 2000; Haering and Rix 2004; McConnell et al. 2012 and references therein, and several contributions in this workshop, e.g. by $\mathrm{Wu}$ ).

The second approach is based on measurements of the instantaneous mass growth rate of $\mathrm{BHs}$ and the stars in their host galaxies. Measuring $\mathrm{BH}$ growth rate is relatively simple, even at very high redshift since it is directly proportional to the bolometric luminosities of the AGN (hereafter $L_{A G N}$ ). The uncertainties here are associated with the somewhat unknown value of the bolometric correction factor requires to convert observed monochromatic luminosities, like $\lambda L_{\lambda}$ at 5100 or $3000 \AA$, to $L_{A G N}$ (see e.g. Trakhtenbrot and Netzer 2012), and the unknown mass to radiation conversion efficiency, $\eta$, in the equation $L_{A G N}=\eta \dot{M} c^{2}$, where $\dot{M}$ is the mass accretion flow into the BH. The stellar mass growth rate is obtained from the star formation rate (SFR) in the host galaxy as measured by one of several well established methods. Here I will focus on SFR estimates which are based on the integrated infrared (IR) luminosity of the galaxy, LIR. I prefer to use the term "star formation luminosity", $L_{S F}$.

Obviously there is a fundamental difference between the integrated and differential methods since galaxies do not disappear from view once they finish a star formation episode while BHs do and their mass cannot be measured for most of the time. The only exceptions are BHs in the local universe where $M_{\mathrm{BH}}$ can be measured by various dynamical methods (see McConnell et al. 2012 and references therein). Unfortunately, such measurements are limited to a small number of local

galaxies and it is not entirely clear whether the objects observed so far represent well the entire population of bulge containing galaxies.

\subsection{Integrated co-evolution: the mass function of BHs}

The integrated co-evolution model requires a complete knowledge of both galaxy and BH MFs at all redshifts. Assume a time $t$ in the history of the universe when the number density of BHs per unit co-moving volume per unit mass $M$ (the "number density mass function") is $N_{a c t}(M, t)$ for active BHs and $N(M, t)$ for all BHs. $N(M, t)$ can be interpreted as the first moment of a more specific distribution function, $g(M, \dot{M}, t)$, that depends on $\mathrm{BH}$ mass $M$, BH accretion rate, $\dot{M}$, and time,

$$
N(M, t)=\int_{0}^{\infty} g(M, \dot{M}, t) d \dot{M} .
$$

The mean accretion rate for a given $\mathrm{BH}$ mass is,

$$
\langle\dot{M}(M, t)\rangle=\frac{1}{N(M, t)} \int_{0}^{\infty} \dot{M} g(M, \dot{M}, t) d \dot{M},
$$

and the MF in units of mass is $\Phi(M, t)=N(M, t) M$. 


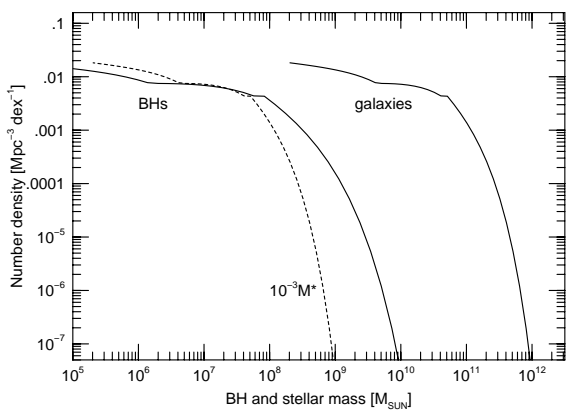

Figure 1: Observed galaxy MF in the local universe (adopted from data published in Li and White (2009) and derived BH MF calculated by the author. A scaled down galaxy MF, by a factor 0.001 , is shown by a dotted line for easy comparison.

It is simple to construct $N_{a c t}(M, t)$ from BH mass measurements and the bolometric luminosity function (LF) of AGNs, $\Phi(L, z)$. Such information is available for a very large number of type-I AGNs up to $z \simeq 2$ and for smaller samples at higher redshifts. BH masses are not available for type-II AGNs with $z \gtrsim 0.3$ and for very faint type-I sources at high redshift.

A general solution for the number density mass function can be obtained by using a continuity argument that relates the value of $N(M, t)$ at times prior to $t$ to its value at time $t$ (e.g. Shankhar et al. 2013). We can use the $\mathrm{BH}$ duty cycle (the fraction of time the $\mathrm{BH}$ is active at time $t$ in the history of the universe), $f_{a c t}(M, t)$, to relate $N(M, t)$ to $\Phi(L, z)$,

$$
\Phi(L, t) d L=f_{\text {act }}(M, t) N(M, t) d M .
$$

The two unknowns in the above approach are $f_{\text {act }}(M, t)$ and its initial value, $f_{\text {act }}\left(M, t_{0}\right)$, where $t_{0}$ is an early time at redshift $z_{0}$ corresponding to the start of the BH growth process. A reasonable estimate is that at very early times, immediately after the formation of the first massive BHs, $f_{\text {act }}\left(M, t_{0}\right)=1$. As shown below, this is now confirmed for the most massive BHs. Unfortunately, much less is known about duty cycles at later time and a direct solution of Eqn. 1.3 cannot be obtained unless we assume the same normalized accretion rate, $L / L_{\text {Edd }}$, for all active BHs. As shown by many observations, this is definitely not the case at all redshifts. There are other fundamental unknowns related to the possible time-dependence of $\eta$ (or BH spin) and the mass dependence of the duty cycle.

The situation at $\mathrm{z}=0$ is different. Here the value of $N(M, 0)$ can be checked against other estimates based on the MF of local galaxies and the known relationship between $M_{\mathrm{BH}}$ and $M_{\text {bulge }}$. Such a comparison is shown in Fig. 1. The galaxy MF in this case was derived by Li and White (2009) for a large number of low redshift SDSS galaxies and the difference in shape from the $\mathrm{z}=0$ $\mathrm{BH} \mathrm{MF}$ at the high mass end reflects the evidence that $M_{\text {bulge }} / M_{\mathrm{BH}}$ is larger for smaller bulges.

Despite the various limitations of the integrated approach, the general conclusions are quite robust. They suggest a scenario where the most massive BHs grew first while the less massive BHs, those with $M_{\mathrm{BH}} \sim 10^{7}-10^{8} M_{\odot}$, started their growth much later and are still growing at a fast rate today. Thus, $\mathrm{BH}$ grow in an anti-hierarchical fashion. Such evolution is similar but not identical to 
the evolution of galaxies. Most importantly, it is not known whether the $\mathrm{BH}$ mass to stellar mass ratio evolves with time.

\subsection{Instantaneous co-evolution: relative growth rate of $\mathrm{BH}$ and stellar mass}

As explained, $L_{A G N}$ and $L_{S F}$ provide information about the relative growth rates of BHs and stellar masses. AGN and SF galaxy surveys, including the large Herschel surveys, provide the empirical information needed for such an analysis. A simple example is the $L_{S F}$ vs. $L_{A G N}$ diagrams for "AGN dominated sources", those where $L_{A G N}>L_{S F}$. The diagram has been studied by Netzer (2009), Shao et al. (2010), Rosario et al. (2012) and others (see Rosario contribution in these proceedings). It suggests $L_{S F} \propto L_{A G N}^{0.7}$, with $L_{S F} \simeq L_{A G N}$ at $L_{A G N} \sim 10^{43} \mathrm{ergs} \mathrm{s}^{-1}$. Recall that the $\mathrm{BH}$ growth rate depends on the efficiency factor $\eta$ (which is known to within a factor of $\sim 2$ ) and the stellar mass growth rate depends on the SFR with its own typical efficiency. We can thus use the data at every point in the $L_{A G N}-L_{S F}$ plane to obtain the ratio $g$ (stellar mass) $/ g(B H)$ where $g$ stands for growth rate,

$$
\frac{g(\text { stellar mass })}{g(B H)} \simeq 140\left[\frac{\eta_{B H} / 0.1}{\eta_{S F} / 7 \times 10^{-4}}\right]\left[\frac{L_{S F}}{L_{A G N}}\right],
$$

Obtaining this information for every point and every redshift provides an efficient toot to study instantaneous co-evolution. It requires two additional pieces of information: the fraction of AGN hosts that are not forming stars at a given redshift, and the fraction of SF galaxies that contain dormant BHs. These, again, are directly related to the duty cycle of both AGNs and SF galaxies.

\section{Is there a mass sequence of active black holes?}

\section{1 $L_{A G N}$ vs. $M_{\mathrm{BH}}$ for AGNs}

Separating galaxies into blue and red groups, or more specifically into SF galaxies and quiescent ("red and dead") galaxies is known to be an efficient tool for probing the SF history of the universe. Perhaps the most effective way to study this evolution is to plot SFRs of individual galaxies against the stellar mass at various redshifts. A clear feature on all such diagrams is the so-called "main sequence" (or better be referred to as the SF sequence or the mass sequence - MS) which is a well define band occupying a relatively narrow region in the diagram and can be expressed as

$$
S F R=a(z) M_{*}^{b} .
$$

Here $a(z)$ is an increasing function of the redshift representing the fact that the relative fraction of gas, mostly molecular gas, is decreasing with cosmic time, and $b \sim 0.6-0.9$. Galaxies that are not forming stars at high rate occupy the region below the MS mostly at the high mass end of the diagram. Most AGN hosts are known to be SF galaxies (see e.g. D. Rosario's contribution) and hence are found on the MS, with a clear concentrations close to the high mass end.

Can similar diagrams be constructed for active BHs? This is a reasonable suggestion given that accretion onto BHs seem to have two distinct modes. High accretion rate $\left(L / L_{\text {Edd }} g t \operatorname{sim} 0.01\right)$ is thought to occur mostly through thin or slim accretion disks. The mass-to-radiation conversion efficiency in such cases $(\eta)$ is very large, typically in the range 0.04-0.3. Radiation inefficient accretion flows (RIAFs), with a much lower conversion efficiency, are thought to power the lower 

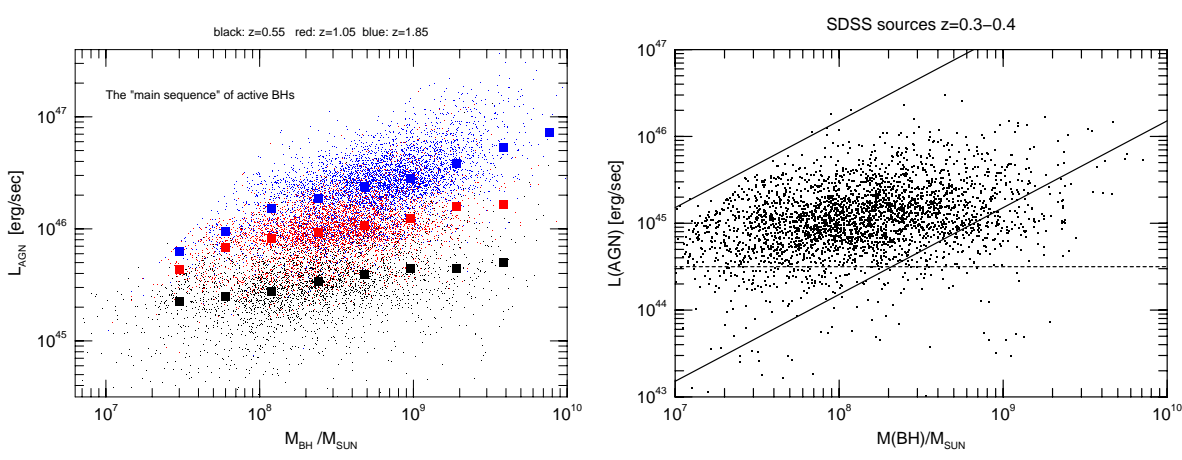

Figure 2: The MS of active BHs: Left: $L_{A G N}$ vs $M_{\mathrm{BH}}$ for three redshift groups, as marked. An apparent sequence is evident with $L_{A G N}$ at a given $M_{\mathrm{BH}}$ increasing with redshift, much like in SF galaxies. Right: A detailed view of the group of AGNs with $z=0.3-0.4$ showing how the flux limit of the sample (SDSS), marked by horizontal line, plus the natural limit of $L / L_{\mathrm{Edd}}=1$ and at $L / L_{\mathrm{Edd}}=0.01$ (top and bottom diagonal lines) give the false impression of a MS.

luminosity LINERs, that outnumber the high efficiency AGNs in the local universe by a large factor. Thus, there are two very different types of accretion that can perhaps be separated in the $L_{A G N}$ vs. $M_{\mathrm{BH}}$ diagram, as blue (SF) and red galaxies occupy different regions in the SFR vs. $M_{*}$ diagram for galaxies. An attempt to construct such a diagram for active BHs is shown in Fig. 2.

\subsection{The era of selection effects}

Fig. 2 seems to resemble the equivalent diagram for galaxies. $L_{A G N}$ clearly increases with $\mathrm{BH}$ mass giving an impression of a MS. Moreover, there is a clear upward shift of the MS at higher redshift. Unfortunately, this behavior is most likely due to the combination of several selection effects that determine the way such object were found. All the sources in the diagram are taken from the Trakhtenbrot and Netzer (2012) sample of SDSS/DR7 sources. This is a flux limited sample and hence, the flux limit dictates the lowest $L_{A G N}$ for a given redshift. The flux limits is translated to higher $L_{A G N}$ at higher redshift which results in the impression that the MS is shifted upward. This is clearly shown in the right hand side of the diagram that shows the flux limit for the group of sources at $z=0.3-0.4$.

We live in an "era of selection effects". Herschel samples at high redshifts suffer from this because of the limited sensitivity of Herschel/PACS, the confusion limit of Herschel/SPIRE, and the differences in integration times between the small (deep) and the large (shallow) Herschel surveys. This may have important implications on the $L_{A G N}$ vs. $L_{S F}$ diagram and must be taken into account in the modeling. Similar selection effects influence several known correlations involving AGNs, mostly because of the lack of large, deep, AGN samples that can probe more accurately the large population of low luminosity AGNs, those with $L / L_{\mathrm{Edd}}$ close to the transition from efficiently accreting BHs to RIAFs.

\section{BH activity star formation and duty cycle at high-z}

While estimating the duty cycle is, in general, a very challenging task, it seems that this issue 
may have been solved for the highest luminosity, most massive BHs at high redshift. The group of sources in question are the most massive BHs at redshifts 2-5, those with $M_{\mathrm{BH}}>10^{9} M_{\odot}$. These objects are drawn from the same population now observed in the centers of the most massive local galaxies, with $M_{\mathrm{BH}} \sim 10^{10} M_{\odot}$ (e.g. McConnell et al 2012). Several groups of such objects were observed, spectroscopically, at redshifts around 2.4, 3.3 and 4.8 where reliable mass estimates, based on the $\mathrm{H} \beta$ and $\mathrm{Mg}$ II $\lambda 2798$ emission lines, can be obtained (see Trakhtebbrot et al. 2011 and references therein). Perhaps the most striking result based on these observations is that the normalized accretion rate, $L / L_{\text {Edd }}$ in this population keep rising with redshift all the way to $z \sim 5$ where it reaches a value very close to unity. Given the measure $M_{\mathrm{BH}}$ and $L_{A G N}$, we can calculate the fraction of time such objects must grow, since their formation at $z=10-20$, in order to explain their mass (i.e. their duty cycle). This fraction is found to be about 1 between $z=10$ and $z=4.8$, and about $0.1-0.2$ between $z=4.8$ and $z=3.3$ and $z=2.4$. These results are shown in Fig. 3 . The known duty cycles provide a critical point to start following the co-evolution of this population provided the parallel information on their SF history is known.

Recently we observed the $\mathrm{z}=4.8$ sample with Herschel/SPIRE and are now in a position to try and compare their SF and AGN history. Part of the observations are described in Mor et al (2012) and part in a soon to be submitted paper (Netzer et al. 2013). The most important results of the work can be summarized as follow:

1. About $25 \%$ of the $\mathrm{z}=4.8$ AGNs are detected by Herschel with extremely high SFR (2000$5000 M_{\odot} y r^{-1}$ assuming a Salpeter IMF), comparable to the SFRs found in the most luminous SMGs.

2. Stacking analysis of the remaining sources results in a clear signal indicating a mean SFR of about $600 M_{\odot} y r^{-1}$.

3. The detected sources all lie above the "AGN dominated" line in the $L_{A G N}$ vs. $L_{S F}$ diagram, roughly on a line where $L_{A G N}=L_{S F}$. The stacked sources are very close to the AGN dominated line for lower redshift AGNs.

There are various way to interpret these results, the most interesting one is related to the suggestion that all detected sources are associated with large mergers (and are hence well above the MS at $z=4.8$ ). Given this suggestion, we may be witnessing a situation that most of the $z=4.8$ AGNs have already finished the main part of their stellar mass accumulation, while some objects are still in the merger phase and hence their stellar mass has not yet reached its maximum value. Fig. 3 shows the combined SED of the detected sources in this sample.

\section{Modeling the $L_{A G N}$ vs. $L_{S F}$ evolution}

Present day theoretical models are limited in their ability to model the complex, redshift dependent co-evolution of BHs and their host galaxies. While N-body simulation are quite successful in following dark matter and halo evolution over a large mass range, this is not the case for the hydrodynamical calculations following the evolution of baryonic matter. There are several realistic estimates of large scale gas flows in halos (even here the various "schools" are in some disagreement) but present day resolution is inadequate to follow SF and bulge built-up in most systems. 

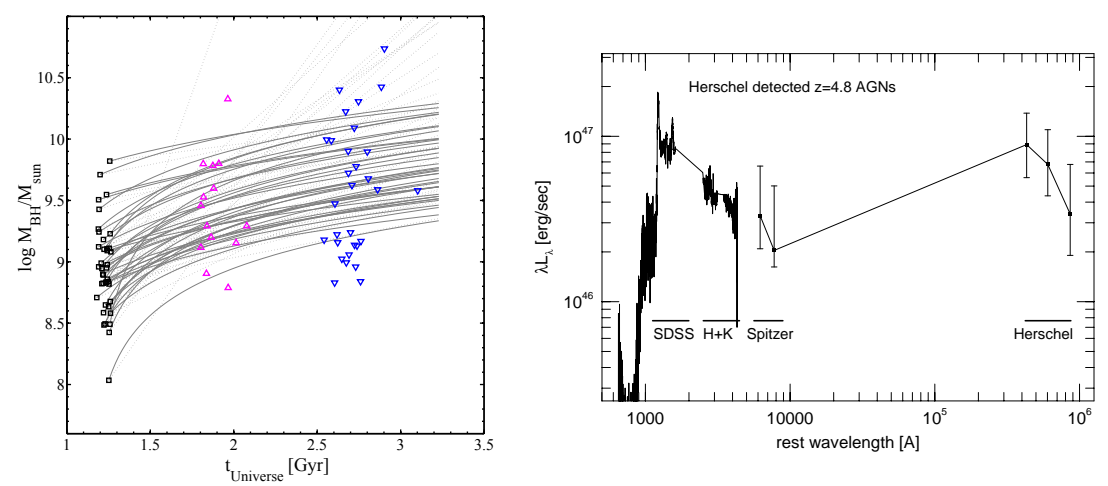

Figure 3: Left: Duty cycle of the most massive BHs at high redshift. The solid lines illustrate the case where $\mathrm{BHs}$ at $\mathrm{z}=4.8$ (black points) grow in a linear way to $\mathrm{z}=3.3$ (magenta) and then to $\mathrm{z}=2.4$ (blue) with a duty cycle of about 0.15 assuming the accretion rate is as measured at $\mathrm{z}=4.8$ and the three groups represent the same parent population (the most massive BH. See Trakhtenbrot et al. 2011). Right: The median UV-FIR SED of the Herschel detected $z=4.8$ AGNs from the sample shown on the left (from Netzer et al. 2013).

Major obstacles are related to the calculations of secular processes, such as disk instabilities, that are essential for explaining the observed SF in individual galaxies. Following the cold gas to the center of the system, and calculating the $\mathrm{BH}$ accretion and growth rate, is way beyond the capabilities of such models.

Semi-analytic models (SAMs) provide a way to combine the detailed N-body simulations, and the limited hydrodynamical calculations, into a single more coherent picture. Such calculations are based on a number of recipes that were tested against the more detailed calculations and the general framework of SAMs allow their application to a very large number of objects. Such recipes can specify the dependence of the SFR on the amount of gas in the disk of the galaxy, the fraction of cold dust that reach the central BH and the efficiency of the accretion process, the outcome of large (similar masses) and small (very different masses) mergers, etc.

In a recent work (Neistein and Netzer 2013) we have followed the co-evolution of SF galaxies and their central BHs using the SAM model of Neistein \& Weinmann (2010) and Wang, Weinmann $\&$ Neistein (2012). The model was developed to follow galaxy evolution thorough time and is very successful in explaining many observations related to galaxies such as their MS at all redshifts, the SFR at $\mathrm{z}=0$ and the galaxy correlation function. We have added $\mathrm{BH}$ evolution to this SAM, focusing on a case where the triggering of $\mathrm{BH}$ accretion is caused only by mergers. The merger can be of similar mass galaxies (mass ratio smaller than about 4:1) in which case the prediction is that signs of the interactions will be found by the observations, or very different masses (mass ratio larger than 4:1) which can trigger $\mathrm{BH}$ accretion without being detected by the observations (and hence be classified as a secular evolving systems). Fig. 4 shows the predicted $L_{A G N}$ vs. SFR relations and the comparison with the Rosario et al. (2012) observations. The SAM can reproduce the observations for a large range of redshifts and provides a simple explanation to this behavior which is related to the much longer duration of the SF events compared with the duration of $\mathrm{BH}$ activity. Secular processes in this model are very important in explaining the stellar mass growth but cannot explain the data related to $\mathrm{BH}$ activity. This kind of calculations can be expanded in the future in attempt to explore other possible scenarios. 


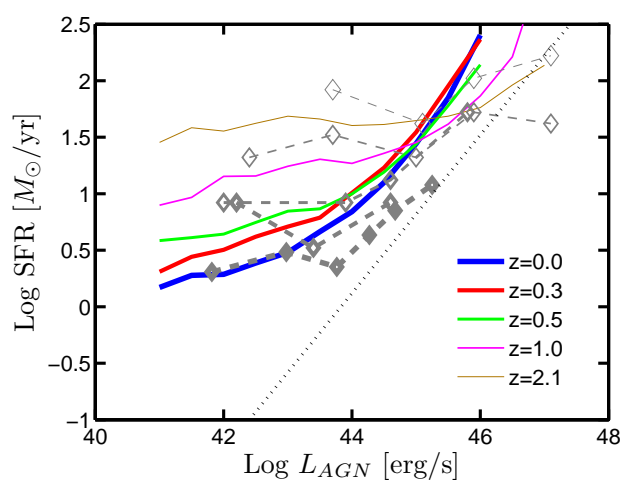

Figure 4: Average SFRs (vertical axis) for the host galaxies AGNs with a given $L_{A G N}$. Data from Rosario et al. (2012) are shown in grey dashed lines, where thinner lines correspond to higher redshifts. Model results using the same averaging scheme and the same redshift bins as used by Rosario et al. are plotted in solid lines with redshifts as indicated. The dotted line shows the relation SFR $\propto \mathrm{L} \beta^{0.7}$

Acknowledgements: I am grateful to my long-term collaborators, Benny Trakhtenbrot, Paulina Lira, Rivay Mor and Ohad Shemmer for their many contributions, ideas and suggestions that helped to shape this review. The part About $L_{A G N}$ vs. $L_{S F}$ was influenced by numerous discussions with Dieter Lutz and David Rosario, and from the detailed SAM calculations of Eyal Neistein.

\section{References}

[1] Ferrarese L., Merritt D., 2000, ApJL, 539, L9

[2] Gebhardt K., et al., 2000, ApJL, 539, L13

[3] Häring N., Rix H.-W., 2004, ApJL, 604, L89

[4] Li, C., \& White, S. D. M. 2009, MNRAS, 398, 2177

[5] McConnell, N. J., Ma, C.-P., Murphy, J. D., et al. 2012, ApJ, 756, 179

[6] Mor R., Netzer H., Trakhtenbrot B., Shemmer O., Lira P., 2012, ApJL, 749, L25

[7] Neistein E., Weinmann S. M., 2010, MNRAS, 405, 2717

[8] Neistein, E. and Netzer, H. 2013 (submitted to MNRAS).

[9] Netzer H., 2009, MNRAS, 399, 1907

[10] Rosario D. J., et al., 2012, A\&A, 545, A45

[11] Shankar, F., Weinberg, D. H., \& Miralda-Escudé, J. 2013, MNRAS, 428, 421

[12] Shao L., et al., 2010, A\&A, 518, L26

[13] Trakhtenbrot, B., Netzer, H., Lira, P., \& Shemmer, O. 2011, ApJ, 730, 7

[14] Trakhtenbrot, B., \& Netzer, H. 2012, MNRAS, 427, 3081

[15] Wang L., Weinmann S. M., Neistein E., 2012, MNRAS, 421, 3450 